\title{
Dopamine transporter single-photon emission computed tomography derived radiomics signature for detecting Parkinson's disease
}

Takuro Shiiba ( $\square$ takuro.shiiba@fujita-hu.ac.jp )

Fujita Health University https://orcid.org/0000-0001-5515-091X

Kazuki Takano

Fujita Health University: Fujita Ika Daigaku

Akihiro Takaki

Teikyo University: Teikyo Daigaku

Shugo Suwazono

National Hospital Organization Okinawa National Hospital

\section{Research Article}

Keywords: Parkinson's disease, dopamine transporter, SPECT, radiomics signature, texture, radiomics

Posted Date: February 9th, 2022

DOI: https://doi.org/10.21203/rs.3.rs-1304269/v1

License: (a) (i) This work is licensed under a Creative Commons Attribution 4.0 International License. Read Full License 


\section{Abstract}

Background: We hypothesised that the radiomics signature, which includes texture information of dopamine transporter single-photon emission computed tomography (DAT-SPECT) images for Parkinson's disease (PD), may assist semi-quantitative indices. Herein, we constructed a radiomics signature using DAT-SPECT-derived radiomics features that effectively discriminated PD from healthy individuals and evaluated its classification performance.

Results: We analysed 291 cases of both normal control $(N C, n=70)$ and PD $(n=221)$ groups from the Parkinson's Progression Markers Initiative database. Data were divided into the training and test sets. DAT-SPECT images were spatially normalised to the Montreal Neurologic Institute space. We calculated 930 radiomics features, including intensity- and texture-based features in the caudate, putamen, and pallidum volumes of interest. The striatum uptake ratios (SURs) of the caudate, putamen, and pallidum were also calculated as conventional semi-quantification indices. The least absolute shrinkage and selection operator was used for feature selection and construction of the radiomics signature. The four classification models were constructed using a radiomics signature and/or semi-quantitative indicator. Furthermore, we compared the classification performance of the semi-quantitative indicator alone and the combination with the radiomics signature for the classification models. The receiver operating characteristics (ROC) analysis was used to evaluate the classification performance. The classification performance of SUR putamen was significantly higher than that of other semi-quantitative indicators. The radiomics signature resulted in a slightly increased area under the ROC curve (AUC) compared to $S_{\text {SUR }}$ putamen in the training and test sets. When combined with SUR putamen and radiomics signature, all classification models showed slightly higher AUCs than that of SUR $\mathrm{Rutamen}_{\text {alone. }}$

Conclusion: We constructed a DAT-SPECT image-derived radiomics signature. Performance analysis showed that the current radiomics signature would be helpful for the diagnosis of PD and has the potential to provide new diagnostic value.

\section{Background}

Parkinson's disease (PD) is characterised by the degeneration of the nigrostriatal dopamine nerve and the appearance of inclusion bodies containing a-synuclein as the main component (i.e. Lewy bodies) [1-3]. The striatum to which dopamine neurones are projected is a nerve nucleus constituting the basal ganglia and comprises the caudate nucleus and putamen. Dopamine transporter (DAT)-single-photon emission computed tomography (SPECT) contributes to the diagnosis of PD and Lewy body dementia by providing a SPECT image reflecting the DAT distribution density in the striatum. Generally, the evaluation of DATSPECT images is conducted via visual inspection, frequently supported by semi-quantitative ratios, such as the striatum uptake ratio (SUR) or specific binding ratio (SBR) [4-7]. In visual assessment, information regarding the asymmetry of the left and right striata and the spatial accumulation site of ${ }^{123}$ I-FP-CIT can be obtained [8-11]. 
Semi-quantitative analysis is hypothesised to eliminate subjectivity and experience differences among readers. Accurate semi-quantitative values may be helpful in the early diagnosis and prediction of the prognosis of PD [12].

Texture analysis [13] can quantitatively represent the heterogeneity of radiopharmaceutical uptake, such as a tumour, in a region of interest [14, 15]. In recent years, radiomics [16-19], which includes texture analysis, is expected to be used not only for diagnosis but also for predicting patient prognosis and determining treatment effects. Texture analysis has also been applied to DAT-SPECT, and texture features correlate with motor and cognitive functions and contribute to the prediction of motor functions [20, 21]. Rahmin et al. [21] showed that Haralick's texture features [22,23] in the caudate nucleus correlated with the Unified Parkinson's Disease Rating Scale and disease duration. Among the large number of texture features, only Haralick's texture features by grey-level co-occurrence matrix were used in these studies. In recent years, many software that can easily calculate radiomics features, including morphology, histogram, and texture, have become widely used for study [24]. However, to our knowledge, constructing a radiomics signature from a wide range of candidate features of DAT-SPECT images and evaluating the classification performance of PD has not been reported. Although the conventional semi-quantitative indices have high classification accuracy $[25,26]$, they do not represent the homogeneity or heterogeneity of radiopharmaceutical distribution in the striatum. The image heterogeneity may become a disturbing factor, which is not well-represented through semi-quantitative indices. Therefore, we hypothesised that the radiomics signature, which includes texture information from DAT-SPECT images, may assist semiquantitative indices. In this study, we constructed a radiomics signature using the radiomics features derived from DAT-SPECT that effectively discriminated PD from healthy individuals and evaluated its classification performance.

\section{Materials And Methods}

\section{Participants}

All data used in this study were obtained from the Parkinson's Progression Markers Initiative (PPMI) database (www.ppmi-info.org/data) accessed on 3 April 2021. The dataset contained 790 pre-processed ${ }^{123}$ I-FP-CIT DAT-SPECT images acquired at the screening stage. In this study, we included cases acquired with the same manufacturer's SPECT system (SIEMENSE) and were continuously enrolled in PPMI from the 790 cases. Table 1 shows the characteristics of the patients in this study. There were 291 patients in both the normal control (NC) and PD groups. The NC and PD group comprised 70 (49 men and 21 women, $61.2 \pm 9.6$ years) and 221 (140 men and 81 women, $60.0 \pm 11.0$ years) individuals, respectively. All data were divided into the training and test sets at 7:3 so that the ratio of the NC and PD groups would be constant. The number of the training and test sets for the NC and PD groups were 49 and 155 and 21 and 66 , respectively.

Table 1 Patient characteristics 


\begin{tabular}{|lll|}
\hline Characteristics & NC & PD \\
\hline Number of patients & 70 & 221 \\
\hline Male/female & $49 / 21$ & $140 / 81$ \\
\hline Age & $61.2 \pm 9.6$ & $60.0 \pm 11.0$ \\
\hline Age of diagnosis & NA & $60.7 \pm 9.5$ \\
\hline Hoehn and Yahr stage & $0.01 \pm 0.1$ & $1.58 \pm 0.5$ \\
\hline MDS-UPDRS $\square$ & $1.0 \pm 1.9$ & $21.8 \pm 9.3$ \\
\hline MoCA & $28.2 \pm 1.1$ & $27.3 \pm 2.3$ \\
\hline
\end{tabular}

MDS-UPDRS Movement Disorder Society-Unified Parkinson's Disease Rating Scale, MoCA Montreal Cognitive Assessment, $N C$ normal control, $P D$ Parkinson's disease, NA not applicable

\section{Reconstruction and spatial normalisation of SPECT images}

Reconstructed DAT-SPECT images were downloaded from the PPMI website. As per PPMI documentation, pre-processing steps were performed at the Institute for Neurodegenerative Disorders and included the following steps: SPECT imaging and reconstruction: SPECT imaging was acquired at each imaging centre as per the PPMI imaging protocol and sent to the institute for neurodegenerative disorders for processing. SPECT raw projection data were imported to a HERMES (Hermes Medical Solutions, Stockholm, Sweden) system for iterative reconstruction. Iterative reconstruction was performed without filtering. The reconstructed files were transferred to the PMOD (PMOD Technologies, Zurich, Switzerland) for subsequent processing. Attenuation correction ellipses were drawn on the images, and a Chang 0 attenuation correction was applied to images utilising a site-specific $\mu$ that was empirically derived from phantom data acquired during site initiation for the trial. Once attenuation correction was completed, a standard Gaussian three-dimensional (3D) 6.0-mm filter was applied.

Then, the DAT-SPECT images were spatially normalised to Montreal Neurologic Institute (MNI) space using statistical parametric mapping (SPM12, Wellcome Trust Centre for Neuroimaging, London, UK) in MATLAB R2021a (version 9.10, The MathWorks, Inc. Massachusetts, USA). DAT-SPECT images were spatially normalised to the MNI-based template of ${ }^{123}$ I-FP-CIT $[27,28]$ using the old normalise function under identical conditions. After spatial normalisation, the radiological technologist with 13-year clinical experience visually assessed for misalignment between DAT-SPECT and the template. The preprocessed images were saved in the Neuroimaging Informatics Technology Initiative format using $91 \times$ $109 \times 91$ isotropic voxels of $2 \mathrm{~mm}$.

\section{Calculation of radiomics features and semi-quantitative indicators}

The automated anatomical labelling atlas (AAL) 3 [29] voxel of interest (VOI) template was used to calculate the radiomics features. The feature calculation VOIs were the caudate nucleus, putamen, and 
pallidum (Fig. 1). The pallidum was added to the VOls to compensate for errors in spatial normalisation. Radiomics features were calculated using Standardized Environment for Radiomics Analysis (SERA) [30-32] worked on MATLAB. One hundred eighty-six image biomarker standardisation initiativestandardised features [24] were calculated using SERA, including 50 first-order features (statistical, histogram, and intensity histogram features) and higher-order136 3D features (Table 2). A total of 558 radiomics features were calculated for the caudate, putamen, and pallidum VOIs. We also calculated the ratio of the caudate to the putamen or pallidum of radiomics features. These totalled 930 radiomics features. Furthermore, the SUR of the caudate nucleus $\left(S U R_{\text {caudate }}\right)$, putamen $\left(S U R_{\text {putamen }}\right)$, and pallidum $\left(S_{\text {pallidum }}\right)$ were calculated as conventional semi-quantification indices. The SUR was calculated using the following formula [33]:

$\operatorname{SUR}(\%)=\frac{C_{\text {striatum }}-C_{\text {background }}}{C_{\text {background }}} \times 100$

where $\mathrm{C}_{\text {striatum }}$ is the average count of the caudate nucleus, putamen, or pallidum and $\mathrm{C}_{\text {background }}$ is the average count of the occipital lobe. In addition, the ratios of the caudate to the putamen or pallidum $\left(\mathrm{CR}_{\text {putamen }}, \mathrm{CR}_{\text {pallidum }}\right)$ were calculated. All the semi-quantitative indices were compared between the NC and PD groups, and receiver operating characteristic (ROC) [34] analysis was performed.

Table 2 Number of radiomics features per region and their family names

\begin{tabular}{|ll|}
\hline Feature family & Number of features \\
\hline Local intensity & 2 \\
\hline Intensity-based statistics & 18 \\
\hline Intensity histogram & 23 \\
\hline Intensity-volume histogram & 7 \\
\hline GLCM & 50 \\
\hline GLRLM & 32 \\
\hline GLZSM & 16 \\
\hline GLDZM & 16 \\
\hline NGTDM & 5 \\
\hline NGLDM & 17 \\
\hline Total & 186 \\
\hline
\end{tabular}

GLCM grey-level co-occurrence matrix, GLRLM grey-level run-length matrix, GLZSM grey-level zone size matrix, GLDZM grey-level distance zone matrix, NGTDM neighbourhood grey tone difference matrix, NGLDM neighbourhood grey-level dependence matrix 


\section{Radiomics feature selection and signature construction}

The least absolute shrinkage and selection operator (LASSO) [35] function in MATLAB was used to select effective features from the radiomics features. All radiomics features were $z$-scored to mean 0 and standard deviation 1.0 before being inputted to LASSO. LASSO permits the estimation and selection of explanatory variables [36,37], that is, radiomics features with non-zero coefficients. For the selection of radiomics features using LASSO, a 10 -fold cross-validation test was conducted using the training set. Furthermore, the linear combination sum of features with non-zero coefficients when the largest lambda value such that the mean square error (MSE) is within one standard error of the minimum MSE was used as the radiomics signature. We compared the classification performance of the radiomics signature and semi-quantitative indicator that showed the highest classification performance.

\section{Classification model construction with radiomics signature and semi-quantitative indicator}

The classification models for the NC and PD groups were constructed using the radiomics signature and/or semi-quantitative indicator. The four classifiers used were support vector machine (SVM), $\mathrm{k}$ nearest neighbour (KNN), linear discriminant analysis (LDA), and decision tree. The main parameters of each classifier were as follows: SVM (BoxConstraint $=1$, KernelScale $=1$, KernelFunction $=$ polynomial [order $=3]$ ), KNN (NumNeighbors $=1$, Distance $=$ minkowski, Exponent $=2)$, LDA $($ Gamma $=0)$, and decision tree (MinLeafSize $=1$, MinParentSize $=10$ ). The features used were radiomics signature alone, semi-quantitative indicator alone, and both. The training set was used to train the classifier, and the performance of each classification model was evaluated using the test set. Classification performance was evaluated using the area under the ROC curve (AUC).

\section{Statistical analyses}

The radiomics signature and SURs in the NC and PD groups were tested for significant differences using the Wilcoxon rank-sum test. ROC analysis was performed using semi-quantitative indicators and radiomics signature. We used the DeLong [38] test to examine the differences in AUCs, and for multiple comparisons, the Bonferroni correction was performed. The sensitivity, specificity, and accuracy of semiquantitative indices and radiomics signature were calculated using the optimal cut-off values determined based on ROC analysis. Differences were considered statistically significant at $P<0.05$. All statistical analyses were performed using RStudio (version 1.4.1106).

\section{Results}

Figure 2 shows the distribution of SURs and CRs in the NC and PD groups. There was a significant difference between the NC and PD groups in all regions of the SURs and CRs $(P<0.001)$.

Figure 3 shows the ROC curves of the semi-quantitative indices in all patients. The AUCs and $95 \%$ confidence interval $(\mathrm{Cl})$ of SURs and CRs in each region were, in order from highest to lowest, as follows: SUR putamen $(0.968,0.952-0.986), \mathrm{CR}_{\text {putamen }}(0.913,0.878-0.947), \operatorname{SUR}_{\text {pallidum }}(0.869,0.821-0.915)$, 
SUR caudate $(0.865,0.818-0.912)$, and $\mathrm{CR}_{\text {palidum }}(0.634,0.567-0.701)$. There was a significant difference between SUR putamen and other SURs or CRs $(P<0.001)$.

Table 3 shows the radiomics features and coefficients selected by LASSO in the training set. The maximum lambda value with MSE within one standard error of the minimum MSE was 0.1601 , and eight radiomics features were selected. The selected radiomics features included five putamen, two pallidum, and one $\mathrm{CR}_{\text {putamen. }}$. Coefficients and radiomics features were used to construct the radiomics signature:

Radiomic signature $=-0.1061 \times$ stat_iqr putamen $+0.0091 \times$ ih_skew $_{\text {putamen }}-0.153 \times$ ihrmad $_{\text {putamen }}$ $-0.1064 \times$ dzm_zdnu putamen $-0.0052 \times$ ngt_contrast $_{\text {putamen }}-0.0017 \times$ ngl_dcnu pallidum $-0.0003 \times$ ngl_dcnu_norm pallidum $\times 0.00068 \times$ stat_rms ${ }_{\text {CRputamen }}$

Table 3

Radiomics features and coefficients selected using LASSO

\begin{tabular}{|lllc|}
\hline Feature family & Feature name (tag name) & Region & Coefficients \\
\hline Statistics & Interquartile range (stat_iqr) & Putamen & -0.1061 \\
\hline $\begin{array}{l}\text { Intensity } \\
\text { histogram }\end{array}$ & Skewness (ih_skew) & Putamen & 0.0091 \\
\hline $\begin{array}{l}\text { Intensity } \\
\text { histogram }\end{array}$ & Robust mean absolute deviation (ihrmad) & Putamen & -0.0153 \\
\hline GLDZM & Zone distance non-uniformity (dzm_zdnu) & Putamen & -0.1064 \\
\hline NGTDM & Contrast (ngt_contrast) & Putamen & -0.0052 \\
\hline NGLDM & Dependence count non-uniformity (ngl_dcnu) & Pallidum & -0.0017 \\
\hline NGLDM & $\begin{array}{l}\text { Dependence count non-uniformity normalised } \\
\text { (ngl_dncu_norm) }\end{array}$ & Pallidum & -0.0003 \\
\hline $\begin{array}{l}\text { Statistics } \\
\text { GLDZM grey-level distance zone matrix, NGTDM neighbourhood grey tone difference matrix, NGLDM } \\
\text { neighbourhood grey-level dependence matrix }\end{array}$ & Caudate/putamen & 0.0068 \\
\hline
\end{tabular}

Figure 4 shows the distribution of radiomics signatures between the NC and PD groups. There was a significant difference between the NC and PD groups $(P<0.001)$.

A comparison of the ROC curves for the radiomics signature and SUR $\mathrm{R}_{\text {putamen }}$ is shown in Figure 5. In the training set, the AUCs of the radiomics signature and SUR putamen $_{\text {were }} 0.981(95 \% \mathrm{Cl}, 0.965-0.997)$ and 0.967 (95\% Cl, 0.947-0.987), respectively ( $P=0.1450)$. In the test set, the AUCs of the radiomics signature and SUR putamen were $0.992(95 \% \mathrm{Cl}, 0.981-1.00)$ and $0.973(95 \% \mathrm{Cl}, 0.939-1.00)$, respectively $(P=0.1924)$. 
Table 4 shows the classification accuracy of the radiomics signature and SUR putamen. The accuracy, sensitivity, and specificity of the radiomics signature and SUR putamen were $96.4 \%, 95.8 \%$, and $98.2 \%$ and $96.9 \%, 97.2 \%$, and $96.0 \%$ and $91.5 \%, 92.3 \%$, and $89.3 \%$ and $95.8 \%, 98.6 \%$, and $88.0 \%$ for the training and test sets, respectively.

Table 4

Classification accuracy of radiomics signature and SUR putamen $_{\text {for the }}$ training and test sets

\begin{tabular}{|lllll|}
\hline & \multicolumn{2}{l}{ Training set } & Test set \\
\cline { 2 - 5 } & $\begin{array}{l}\text { Radiomics } \\
\text { signature }\end{array}$ & SUR $_{\text {putamen }}$ & $\begin{array}{l}\text { Radiomics } \\
\text { signature }\end{array}$ & SUR $_{\text {putamen }}$ \\
\hline Accuracy (\%) & 96.4 & 91.5 & 96.9 & 95.8 \\
\hline Sensitivity (\%) & 95.8 & 92.3 & 97.2 & 98.6 \\
\hline Specificity (\%) & 98.2 & 89.3 & 96.0 & 88.0 \\
\hline SUR striatum uptake ratio & & & \\
\hline
\end{tabular}

Table 5 shows the AUCs of each classification model when radiomics signature alone, SUR putamen $_{\text {alone, }}$ and both features were combined. There were no significant differences in the AUCs between SUR putamen alone and in combination with SUR $\mathrm{R}_{\text {putamen }}$ and radiomics signature. However, the AUC was better in all models when combined with SUR $\mathrm{R}_{\text {putamen }}$ and radiomics signature compared to SUR $\mathrm{Putamen}_{\text {alone. }}$ 
Table 5

Classification performance of various classification models using radiomics signature and SUR $\mathrm{R}_{\text {putamen }}$

\begin{tabular}{|c|c|c|c|c|}
\hline \multirow[t]{2}{*}{ Classifier } & \multicolumn{3}{|c|}{ Features AUC (95\% Cl) } & \multirow{2}{*}{$\begin{array}{l}\text { P-value } \\
\text { (SUR } \text { putamen VS }_{\text {combination) }}\end{array}$} \\
\hline & $\begin{array}{l}\text { Radiomics } \\
\text { signature }\end{array}$ & SUR $_{\text {putamen }}$ & Combination & \\
\hline \multirow[t]{2}{*}{ SVM } & 0.992 & 0.976 & 0.997 & \multirow[t]{2}{*}{0.1335} \\
\hline & $(0.968-0.999)$ & $\begin{array}{l}(0.924- \\
0.997)\end{array}$ & $\begin{array}{l}(0.980- \\
1.000)\end{array}$ & \\
\hline \multirow[t]{2}{*}{ KNN } & 0.981 & 0.958 & 0.974 & \multirow[t]{2}{*}{0.1182} \\
\hline & $(0.937-0.999)$ & $\begin{array}{l}(0.876- \\
0.990)\end{array}$ & $\begin{array}{l}(0.888- \\
0.998)\end{array}$ & \\
\hline \multirow[t]{2}{*}{ LDA } & 0.992 & 0.973 & 0.989 & \multirow[t]{2}{*}{0.1153} \\
\hline & $(0.970-0.999)$ & $\begin{array}{l}(0.916- \\
0.997)\end{array}$ & $\begin{array}{l}(0.955- \\
0.999)\end{array}$ & \\
\hline \multirow{2}{*}{$\begin{array}{l}\text { Decision } \\
\text { tree }\end{array}$} & 0.917 & 0.894 & 0.933 & \multirow[t]{2}{*}{0.5432} \\
\hline & $(0.821-0.970)$ & $\begin{array}{l}(0.726- \\
0.981)\end{array}$ & $\begin{array}{l}(0.834- \\
0.980)\end{array}$ & \\
\hline
\end{tabular}

\section{Discussion}

In this study, we constructed and evaluated the potential of a radiomics signature derived from DATSPECT images to classify the NC and PD groups.

The main findings of this study are as follows. First, radiomics signature may have a higher classification performance than semi-quantitative indicators. Second, the combination of radiomics signature and semi-quantitative indicator as features for the classification models would improve the classification performance compared to that of the semi-quantitative indicator alone.

SUR $_{\text {putamen }}$ showed the highest classification performance among the semi-quantitative indices for each region obtained from spatially normalised DAT-SPECT images. ${ }^{123}$-FP-CIT decline began in the putamen in patients with PD, but accumulation was maintained in the caudate of both the PD and NC groups.

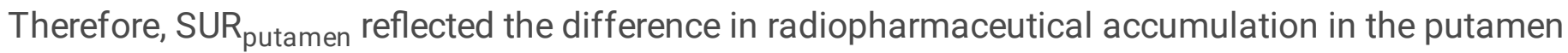
between the NC and PD groups and showed high classification performance. The same reasoning can explain the high classification performance of $\mathrm{CR}_{\text {putamen. }}$. The high classification performance of the semi-quantitative indicator of the putamen is consistent with those of several previous studies [39-41]. It 
is hypothesised that the SUR was higher in the NC group and lower in the PD group due to the inclusion of putamen counts in the pallidum region.

For radiomics feature selection, the most common region to which the eight features selected by LASSO belonged to the putamen, followed by the pallidum. This is because radiopharmaceuticals accumulate less from the putamen in PD, similar to the above. Radiomics features reflect the heterogeneity of radiopharmaceutical accumulations in VOIs. GLDZM (zone distance non-uniformity; tag name, dzm_zdnu $u_{\text {putamen }}$ ), which had the most significant coefficient, is a matrix that shows how far the connected regions with the same concentration value are from the edge of the region of interest. The dzm_zdnu putamen measures the distribution of zone counts over the different zone distances and is low when the zone counts are equally distributed along with the zone distances. In the putamen region, dzm_zdnu putamen in the NC group was significantly higher than that in the PD group (data not shown). This result indicates that the heterogeneity at the edge of the VOI was higher in the NC group and lower in the PD group. This may be because the accumulation of radiopharmaceuticals in the centre and edges of the putamen region differs in the NC group due to the partial volume effect. In contrast, the accumulation of radiopharmaceuticals in the putamen region was uniformly reduced in the PD group. Based on the results of the semi-quantitative indicator and radiomics features, the amount of radioactivity and texture information of the putamen can be a powerful indicator of the differentiation of PD. In this study, VOI settings based on the AAL label images were applied on DAT-SPECT images after spatial normalisation. Because this process does not involve human work, high repeatability and reproducibility can be expected. However, if the spatial normalisation is not performed correctly, the correct radiomics features will not be calculated. Therefore, visual confirmation by an experienced person is required. In this study, no apparent failure of spatial normalisation was visually identified. Furthermore, the semi-quantitative indicators and radiomics features showed high classification performance for the NC and PD groups, suggesting that an appropriate VOI was set.

The radiomics signature showed a higher classification performance between the PD and NC groups than

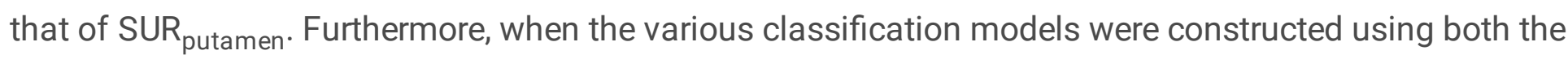
radiomics signature and $S_{\text {SUtamen }}$ as features, the classification performance was better than that of $\mathrm{SUR}_{\text {putamen }}$ alone. This result suggests that the radiomics signature provides robust texture information to supplement the semi-quantitative indicators. Iwabuchi et al. [42] reported that the combined diagnostic accuracy of the three types of indices, SBR, putamen-to-caudate-ratio (PCR), and fractal dimension (or asymmetry index), used for SVM improves diagnostic accuracy. Generally, semi-quantitative indicators assess the quantity of radiopharmaceuticals, and their spatial distribution depends on visual assessment. Adding an indicator for the radiopharmaceutical spatial distribution (e.g. texture information, $\mathrm{PCR}$, fractal dimension) to the semi-quantitative indicator would improve the diagnostic accuracy. We believe that the combination of the semi-quantitative indicator and radiomics signature would lead to the development of highly accurate automatic diagnosis or diagnostic assistant models. 
This study had some limitations. First, we employed a single-manufacturing SPECT system to exclude the influence of the differences in SPECT image quality among manufacturers. Consequently, there was an imbalance between the NC and PD groups. Therefore, it is necessary to investigate an increase in the number of patients. Second, wavelet features were not included in the radiomics features. Because SERA does not support wavelet analysis, other software should be used. Finally, we did not consider differences in the striatum laterally based on a previous report that showed lower accuracy for the asymmetry index than that of SBR and PCR [25]. As lateral differences might be useful for distinguishing between PD in the early stage and other Parkinsonism, such as progressive supranuclear palsy of the parkinsonism subtype [43], signature construction is required.

\section{Conclusions}

In conclusion, the radiomics signature derived from DAT-SPECT images could help distinguish between $\mathrm{NC}$ and PD. Furthermore, the classification performance of various classification models was improved using both radiomics signature and semi-quantitative indicator. Therefore, a radiomics signature, which includes texture information, could provide new diagnostic value when used with semi-quantitative indicators.

\section{Abbreviations}

dopamine transporter single-photon emission computed tomography (DAT-SPECT)

Parkinson's disease (PD)

normal control (NC)

striatum uptake ratio (SUR)

receiver operating characteristics $(\mathrm{ROC})$

area under the ROC curve (AUC)

specific binding ratio (SBR)

three-dimensional (3D)

Montreal Neurologic Institute (MNI)

automated anatomical labelling atlas (AAL)

voxel of interest ( $\mathrm{VOI})$

Standardized Environment for Radiomics Analysis (SERA) 
SUR of the caudate nucleus (SUR caudate )

SUR of the putamen (SUR putamen $)$

SUR of the pallidum $\left(S_{\text {pallidum }}\right)$

ratio of the caudate to the putamen ( $\left.\mathrm{CR}_{\text {putamen }}\right)$

ratio of the caudate to the pallidum $\left(\mathrm{CR}_{\text {pallidum }}\right)$

least absolute shrinkage and selection operator (LASSO)

mean square error (MSE)

support vector machine (SVM)

k-nearest neighbour (KNN)

linear discriminant analysis (LDA)

confidence interval $(\mathrm{Cl})$

putamen-to-caudate-ratio (PCR)

\section{Declarations}

\section{Ethics Approval and Consent to Participate}

All the clinical and imaging data acquired in this study were conducted in accordance with the Declaration of Helsinki after the approval of the local ethics committees of the sites participating in the PPMI. The relevant local institutional review boards approved the PPMI protocol, and written informed consent was obtained from all patients prior to inclusion. Additional approval was obtained from the Ethics Committee of Fujita Health University (HM21-074) for data analysis.

\section{Consent for publication}

Not applicable

\section{Availability of data and materials}

The datasets analysed during the current study are available in the PPMI repository, https://www.ppmiinfo.org.

\section{Competing Interests}

The authors declare that they have no competing interests. 


\section{Funding}

This work was supported by the JSPS KAKENHI (Grant Number: 18K15565 and 21K15791).

\section{Author information}

Affiliations

Faculty of Radiological Technology, School of Medical Sciences, Fujita Health University, 1-98, Dengakubo, Kutsukake-cho, Toyoake, Aichi, 470-1192, Japan

Takuro Shiiba and Kazuki Takano

Department of Radiological Technology, Faculty of Fukuoka Medical Technology, Teikyo University, 6-22 Misakimachi, Omuta-shi, Fukuoka 836-8505, Japan

Akihiro Takaki

Department of Neurology and Center for Clinical Neuroscience, National Hospital Organization Okinawa National Hospital, 3-20-14 Ganeko, Ginowan, 901-2214, Japan

Shugo Suwazono

\section{Author's contributions}

All authors contributed to the conception and design of the study. Material preparation, data collection, and analysis were performed by TS and KT. Spatially normalised SPECT was visually assessed by TS. TS wrote the original draft of the manuscript, and KT, AT, SS wrote the review and edited the previous versions of the manuscript. All authors have read and approved the final manuscript.

\section{Acknowledgements}

Data used in the preparation of this article were obtained from the PPMI database. PPMI - a publicprivate partnership - is funded by the Michael J. Fox Foundation for Parkinson's Research funding partners 4D Pharma, Abbvie, Acurex Therapeutics, Allergan, Amathus Therapeutics, ASAP, Avid Radiopharmaceuticals, Bial Biotech, Biogen, BioLegend, Bristol-Myers Squibb, Calico, Celgene, Dacapo Brain Science, Denali, The Edmond J. Safra Foundaiton, GE Healthcare, Genentech, GlaxoSmithKline, Golub Capital, Handl Therapeutics, Insitro, Janssen Neuroscience, Lilly, Lundbeck, Merck, Meso Scale Discovery, Neurocrine Biosciences, Pfizer, Piramal, Prevail, Roche, Sanofi Genzyme, Servier, Takeda, Teva, UCB, Verily, and Voyager Therapeutics

\section{References}


1. Tolosa E, Wenning G, Poewe W. The diagnosis of Parkinson's disease. Lancet Neurol [Internet]. 2006;5:75-86. https://doi.org/10.1016/S1474-4422(05)70285-4.

2. Booth TC, Nathan M, Waldman AD, Quigley AM, Schapira AH, Buscombe J. The role of functional dopamine-transporter SPECT imaging in parkinsonian syndromes, part 1. AJNR Am J Neuroradiol. 2015;36:229-35. https://doi.org/10.3174/ajnr.A3970.

3. Booth TC, Nathan M, Waldman AD, Quigley AM, Schapira AH, Buscombe J. The role of functional dopamine-transporter SPECT imaging in parkinsonian syndromes, part 2. AJNR Am J Neuroradiol. 2015;36:236-44. https://doi.org/10.3174/ajnr.A3971.

4. Darcourt J, Booij J, Tatsch K, Varrone A, Vander Borght T, Kapucu ÖL, et al. EANM procedure guidelines for brain neurotransmission SPECT using123I-labelled dopamine transporter ligands, version 2. Eur J Nucl Med Mol Imaging. 2010;37:443-50. https://doi.org/10.1007/s00259-009-1267$\mathrm{x}$.

5. Tossici-Bolt L, Hoffmann SMA, Kemp PM, Mehta RL, Fleming JS. Quantification of [123I]FP-CIT SPECT brain images: an accurate technique for measurement of the specific binding ratio. Eur $\mathrm{J}$ Nucl Med Mol Imaging. 2006;33:1491-9. https://doi.org/10.1007/s00259-006-0155-x.

6. Benamer HTS, Patterson J, Wyper DJ, Hadley DM, Macphee GJ, Grosset DG. Correlation of Parkinson 's disease severity and duration with I-FP-CIT SPECT striatal uptake. Mov Disord. 2000;15:692-8. https://doi.org/10.1002/1531-8257(200007)15:4<692::aid-mds1014>3.0.co;2-v.

7. Bonab AA, Fischman AJ, Alpert NM. Comparison of 4 methods for quantification of dopamine transporters by SPECT with [123I]IACFT. J Nucl Med. 2000;41:1086-92.

8. Kahraman D, Eggers C, Schicha H, Timmermann L, Schmidt M. Visual assessment of dopaminergic degeneration pattern in 123I-FP-CIT SPECT differentiates patients with atypical parkinsonian syndromes and idiopathic Parkinson's disease. J Neurol. 2012;259:251-60. https://doi.org/10.1007/s00415-011-6163-1.

9. Habraken JB, Booij J, Slomka P, Sokole EB, van Royen EA. Quantification and visualization of defects of the functional dopaminergic system using an automatic algorithm. J Nucl Med. 1999;40:1091-7.

10. Eggers C, Kahraman D, Fink GR, Schmidt M, Timmermann L. Akinetic-rigid and tremor-dominant Parkinson's disease patients show different patterns of FP-CIT single photon emission computed tomography. Mov Disord. 2011;26:416-23. https://doi.org/10.1002/mds.23468.

11. Lloyd JJ, Petrides G, Donaghy PC, Colloby SJ, Attems J, O’Brien JT, Roberts G, Thomas AJ. A new visual rating scale for loflupane imaging in Lewy body disease. Neuroimage Clin. 2018;20:823-29. https://doi.org/10.1016/j.nicl.2018.09.012.

12. Bajaj N, Hauser RA, Grachev ID. Clinical utility of dopamine transporter single photon emission CT (DaT-SPECT) with (123I) ioflupane in diagnosis of parkinsonian syndromes. J Neurol Neurosurg Psychiatry. 2013;84:1288-95. https://doi.org/10.1136/jnnp-2012-304436.

13. Ojala T, Pietikäinen $M$, Harwood D. A comparative study of texture measures with classification based on feature distributions. Pattern Recognit. 1996;29:51-9. https://doi.org/10.1016/00313203(95)00067-4. 
14. Orlhac F, Soussan M, Maisonobe JA, Garcia CA, Vanderlinden B, Buvat I. Tumor texture analysis in 18F-FDG PET: relationships between texture parameters, histogram indices, standardized uptake values, metabolic volumes, and total lesion glycolysis. J Nucl Med. 2014;55:414-22. https://doi.org/10.2967/jnumed.113.129858.

15. Chen S, Harmon S, Perk T, Li X, Chen M, Li Y, Jeraj R. Diagnostic classification of solitary pulmonary nodules using dual time F-FDG PET/CT image texture features in granuloma-endemic regions. Sci Rep. 2017;7:1-8. https://doi.org/10.1038/s41598-017-08764-7.

16. van Timmeren JE, Cester D, Tanadini-Lang S, Alkadhi H, Baessler B. Radiomics in medical imaging -"how-to" guide and critical reflection. Insights Imaging. 2020;11:91. https://doi.org/10.1186/s13244-020-00887-2.

17. Schernberg A, Orlhac F, Sun R, Chargari C, Dercle L, Deutsch E, Buvat I, Robert C. Radiomics in nuclear medicine applied to radiation therapy: methods, pitfalls, and challenges. Int $\mathrm{J}$ Radiat Oncol Biol Phys. 2018;102:1117-42. https://doi.org/10.1016/j.jirobp.2018.05.022.

18. Vallières $M$, Freeman $C$, Skamene $S$, El Naqa. A radiomics model from joint FDG-PET and MRI texture features for the prediction of lung metastases in soft-tissue sarcomas of the extremities. Phys Med Biol. 2015;60:5471-96. https://doi.org/10.1088/0031-9155/60/14/5471.

19. Lambin P, Rios-Velazquez E, Leijenaar R, Carvalho S, van Stiphout RGPM, Granton P, et al. Radiomics: extracting more information from medical images using advanced feature analysis. Eur $\mathrm{J}$ Cancer. 2012;48:441-6. https://doi.org/10.1016/j.ejca.2011.11.036.

20. Rahmim A, Huang P, Shenkov N, Fotouhi S, Davoodi-Bojd E, Lu L, et al. Improved prediction of outcome in Parkinson's disease using radiomics analysis of longitudinal DAT SPECT images. Neuroimage Clin. 2017;16:539-44. https://doi.org/10.1016/j.nicl.2017.08.021.

21. Rahmim A, Salimpour Y, Jain S, Blinder S, Klyuzhin I, Smith G, et al. Application of texture analysis to DaTscan images for enhanced assessment of progression in Parkinson's disease. J Nucl Med. 2016;57(supplement2):73.

22. Haralick RM, Dinstein I, Shanmugam K. Textural features for image classification. IEEE Trans Syst Man Cybern. 1973;6:610-21.

23. Haralick RM. Statistical and structural approaches to texture. Proc IEEE. 1979;67:786-804. https://doi.org/10.1109/PROC.1979.11328.

24. Zwanenburg A, Vallières M, Abdalah MA, Aerts HJWL, Andrearczyk V, Apte A, at al. The Image Biomarker Standardization Initiative: standardized quantitative radiomics for high-throughput imagebased phenotyping. Radiology [Internet]. 2020;295:328-38.

https://doi.org/10.1148/radiol.2020191145.

25. Iwabuchi Y, Nakahara T, Kameyama M, Yamada Y, Hashimoto M, Matsusaka Y, at al. Impact of a combination of quantitative indices representing uptake intensity, shape, and asymmetry in DAT SPECT using machine learning: comparison of different volume of interest settings. EJNMMI Res. 2019;9:7. https://doi.org/10.1186/s13550-019-0477-x. 
26. Wenzel M, Milletari F, Krüger J, Lange C, Schenk M, Apostolova I, ea al. Automatic classification of dopamine transporter SPECT: deep convolutional neural networks can be trained to be robust with respect to variable image characteristics. Eur J Nucl Med Mol Imaging. 2019;46:2800-11. https://doi.org/10.1007/s00259-019-04502-5.

27. Salas-Gonzalez D, Górriz JM, Ramírez J, Illán IA, Lang EW. Linear intensity normalization of FP-CIT SPECT brain images using the a-stable distribution. Neurolmage. 2013;65:449-55. https://doi.org/10.1016/j.neuroimage.2012.10.005.

28. Salas-Gonzalez D, Górriz JM, Ramírez J, Illán IA, Padilla P, Martínez-Murcia FJ, et al. Building a FP-CIT SPECT brain template using a posterization approach. Neuroinformatics. 2015;13:391-402. https://doi.org/10.1007/s12021-015-9262-9.

29. Rolls ET, Huang CC, Lin CP, Feng J, Joliot M. Automated anatomical labelling atlas 3. Neurolmage. 2020;206:116189. https://doi.org/10.1016/j.neuroimage.2019.116189.

30. Ashrafinia S. Quantitative nuclear medicine imaging using advanced image reconstruction and radiomics. ProQuest Dissertations and Theses. 2019.

31. Feliciani G, Mellini L, Carnevale A, Sarnelli A, Menghi E, Piccinini F, et al. The potential role of MR based radiomic biomarkers in the characterization of focal testicular lesions. Sci Rep. 2021;11:3456. https://doi.org/10.1038/s41598-021-83023-4.

32. Du D, Feng H, Lv W, Ashrafinia S, Yuan Q, Wang Q, et al. Machine learning methods for optimal radiomics-based differentiation between recurrence and inflammation: application to nasopharyngeal carcinoma post-therapy PET/CT images. Mol Imaging Biol. 2020;22:730-38. https://doi.org/10.1007/s11307-019-01411-9.

33. Crespo C, Gallego J, Cot A, Falcón C, Bullich S, Pareto D, Aguiar P, Sempau J, Lomeña F, Calviño F, Pavía J, Ros D. Quantification of dopaminergic neurotransmission SPECT studies with 123I-labelled radioligands. A comparison between different imaging systems and data acquisition protocols using Monte Carlo simulation. Eur J Nucl Med Mol Imaging. 2008;35:1334-42. https://doi.org/10.1007/s00259-007-0711-z.

34. Hanley JA, McNeil BJ. The meaning and use of the area under a receiver operating characteristic (ROC) curve. Radiology. 1982;143:29-36. https://doi.org/10.1148/radiology.143.1.7063747.

35. Tibshirani R. The lasso method for variable selection in the cox model. Stat Med. 1997;16:385-95. https://doi.org/10.1002/(sici)1097-0258(19970228)16:4<385::aid-sim380>3.0.co;2-3.

36. Huang YQ, Liang CH, He L, Tian J, Liang CS, Chen X, et al. Development and validation of a radiomics nomogram for preoperative prediction of lymph node metastasis in colorectal cancer. $J$ Clin Oncol. 2016;34:2157-64. https://doi.org/10.1200/JC0.2015.65.9128.

37. Wang H, Wang L, Lee EH, Zheng J, Zhang W, Halabi S, et al. Decoding COVID-19 pneumonia: comparison of deep learning and radiomics CT image signatures. Eur J Nucl Med Mol Imaging. 2021;48:1478-86. https://doi.org/10.1007/s00259-020-05075-4.

38. DeLong ER, DeLong DM, Clarke-Pearson DL. Comparing the areas under two or more correlated receiver operating characteristic curves: a nonparametric approach. Biometrics. 1988;44:837-45. 
39. Cuberas-Borrós G, Lorenzo-Bosquet C, Aguadé-Bruix S, Hernández-Vara J, Pifarré-Montaner P, Miquel F, et al. Quantitative evaluation of striatal I-123-FP-CIT uptake in essential tremor and parkinsonism. Clin Nucl Med. 2011;36:991-6. https://doi.org/10.1097/RLU.0b013e3182291a7b.

40. Seibyl JP, Marchek KL, Quinlan D, Sheff K, Zoghbi S, Zea-Ponce Y, et al. Decreased single-photon emission computed tomographic $\{123 \mid\} \beta-C I T$ striatal uptake correlates with symptom severity in parkinson's disease. Ann Neurol. 1995;38:589-98. https://doi.org/10.1002/ana.410380407.

41. Palumbo B, Fravolini ML, Buresta T, Pompili F, Forini N, Nigro P, et al. Diagnostic accuracy of parkinson disease by support vector machine (SVM) analysis of 123I-FP-CIT brain SPECT data. Med (Baltim). 2014;93:e228. https://doi.org/10.1097/MD.0000000000000228.

42. Iwabuchi Y, Nakahara T, Kameyama M, Yamada Y, Hashimoto M, Matsusaka Y, et al. Impact of a combination of quantitative indices representing uptake intensity, shape, and asymmetry in DAT SPECT using machine learning: comparison of different volume of interest settings. EJNMMI Res. 2019;9:7. https://doi.org/10.1186/s13550-019-0477-x.

43. Shigekiyo T, Arawaka S. Laterality of specific binding ratios on DAT-SPECT for differential diagnosis of degenerative parkinsonian syndromes. Sci Rep. 2020;10:15761. https://doi.org/10.1038/s41598020-72321-y.

\section{Figures}

\section{Figure 1}

An example of settings of voxel of interests for calculating radiomics features and semi-quantitative indices

The upper row indicates after the spatial normalisation dopamine transporter single-photon emission computed tomography, and the middle and lower row indicate fused image. The coloured area of the middle and lower rows indicates the striatum and background (occipital lobe). 

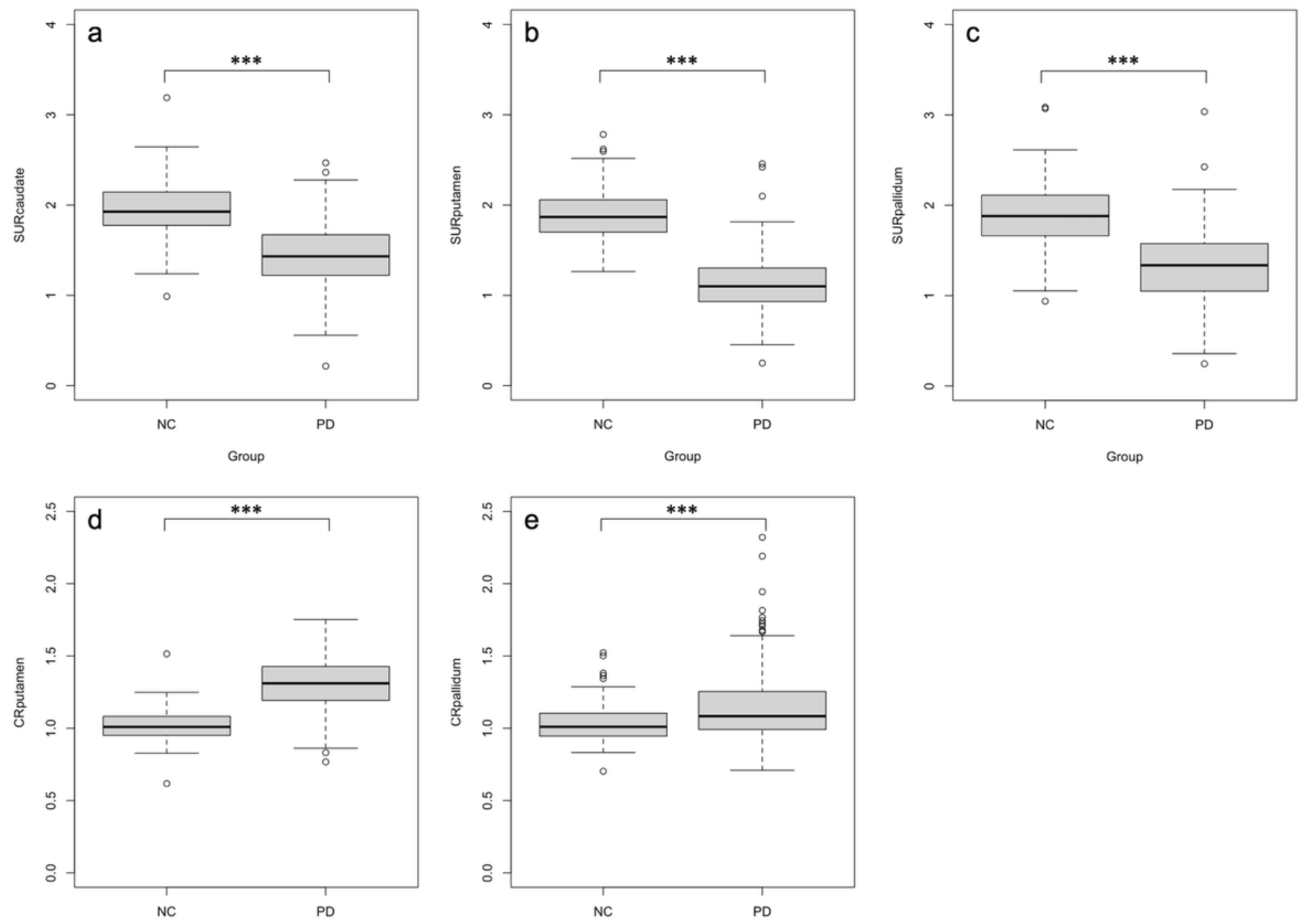

Figure 2

Striatum uptake ratio (SUR) and caudate ratio (CR) between the normal control and Parkinson's disease Box-and-whisker plots indicate the semi-quantitative indices distribution. a SUR $\mathrm{R}_{\text {caudate }}, \mathbf{b} \mathrm{SUR}_{\text {putamen, }} \mathbf{c}$ SUR pallidum; d, $\mathrm{CR}_{\text {putamen, }}$ e $\mathrm{CR}_{\text {pallidum. }}{ }^{* \star *} \mathrm{P}<0.001$ 


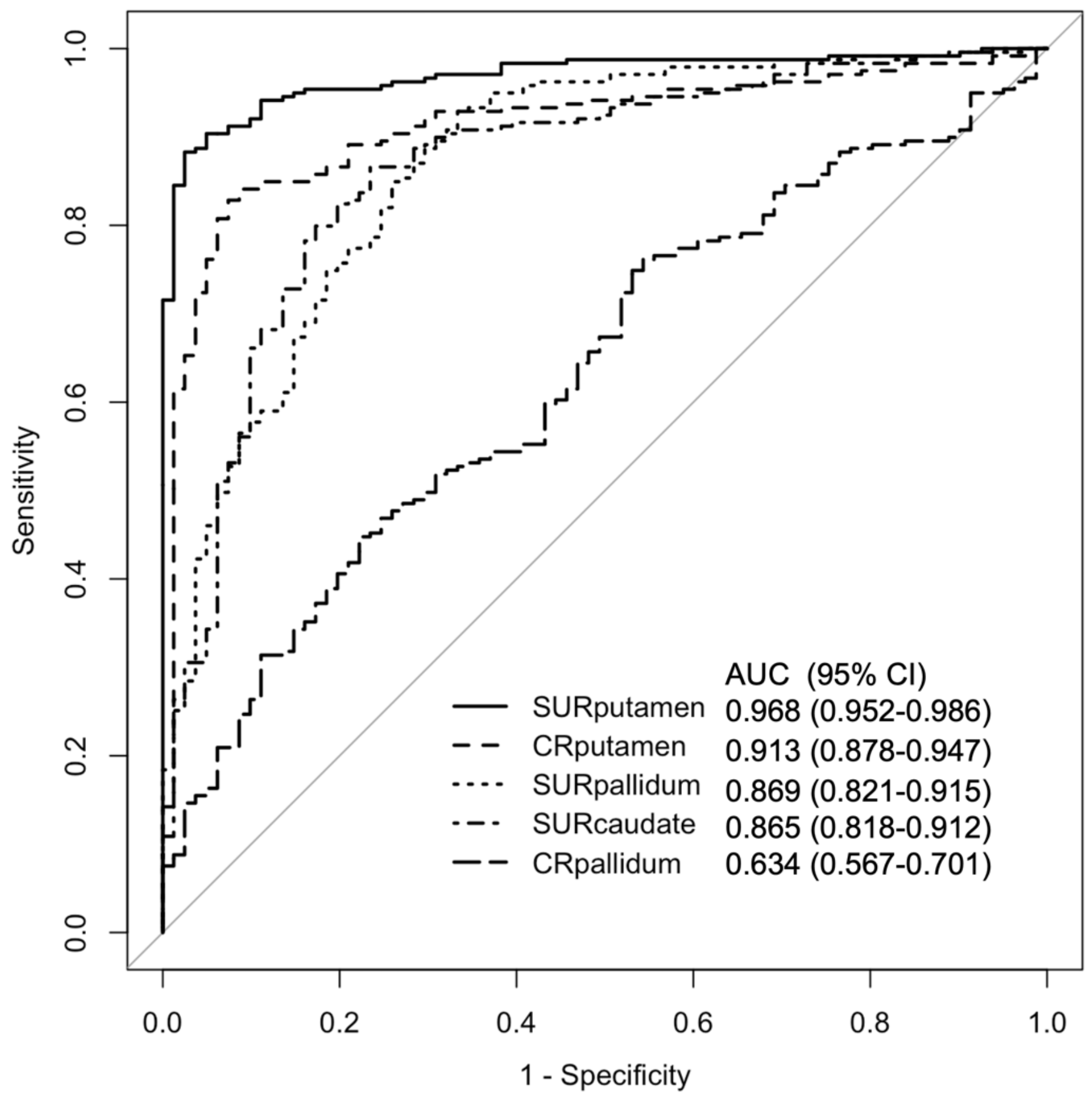

Figure 3

Receiver operating curves for semi-quantitative indices

The AUCs and $95 \%$ confidence interval $(\mathrm{Cl})$ of semi-quantitative indices in each region were SUR $_{\text {putamen }}$ (0.968, 0.952-0.986), CR putamen $(0.913,0.878-0.947)$, SUR $_{\text {pallidum }}(0.869,0.821-0.915)$, SUR $_{\text {caudate }}$ $(0.865,0.818-0.912)$, and $\mathrm{CR}_{\text {palidum }}(0.634,0.567-0.701)$. There was a significant difference between SUR $_{\text {putamen }}$ and other semi-quantitative indices $(P<0.001)$. 
$A U C$ area under the curve, $C /$ confidence interval, SUR striatum uptake ratio, $C R$ caudate ratio
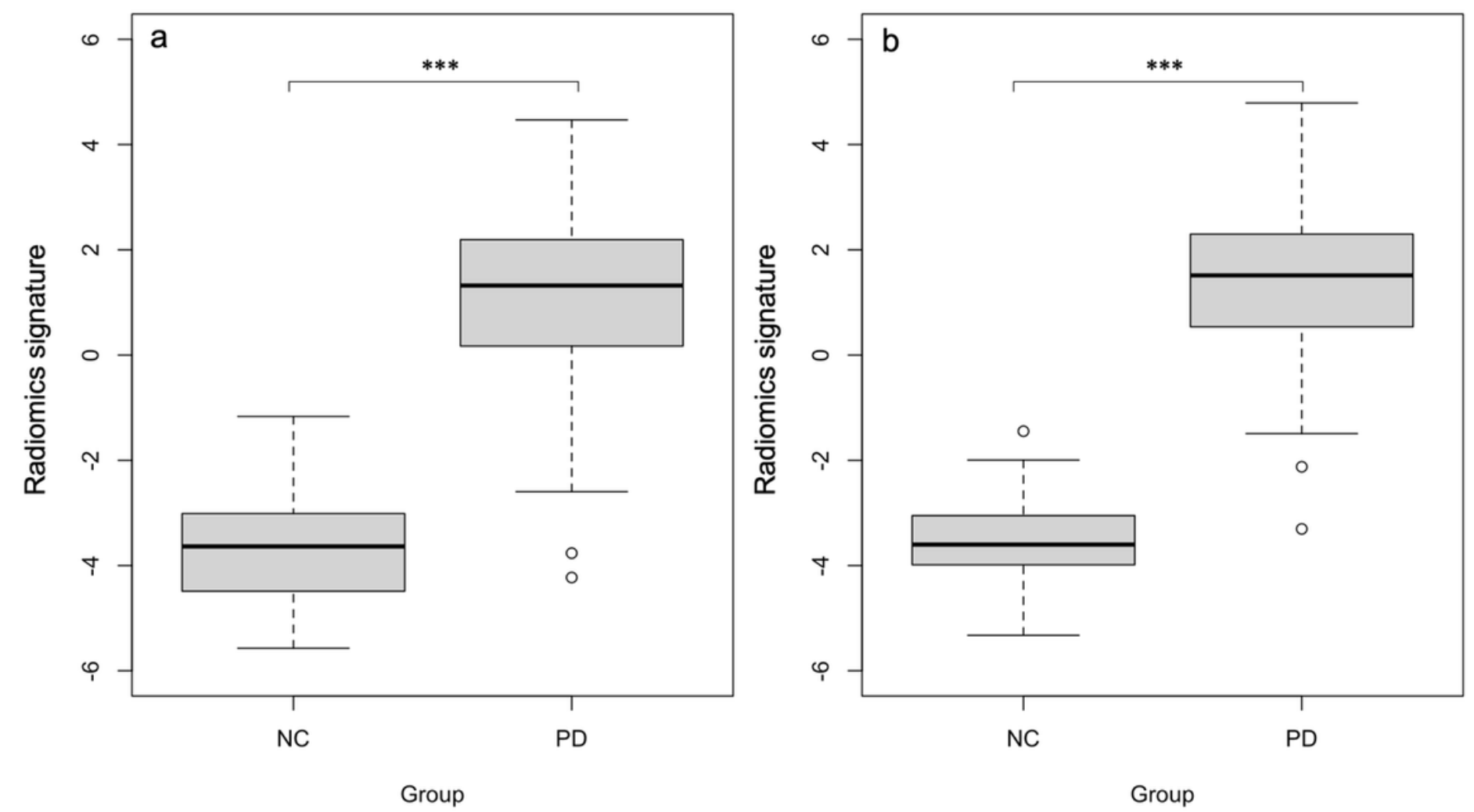

\section{Figure 4}

Comparison of radiomics signature between the normal control and Parkinson's disease Box-and-whisker plots indicates the radiomics signature distribution. a training set, $\mathbf{b}$ test set. ${ }^{* \star} \mathrm{P}<$ 0.001 
a

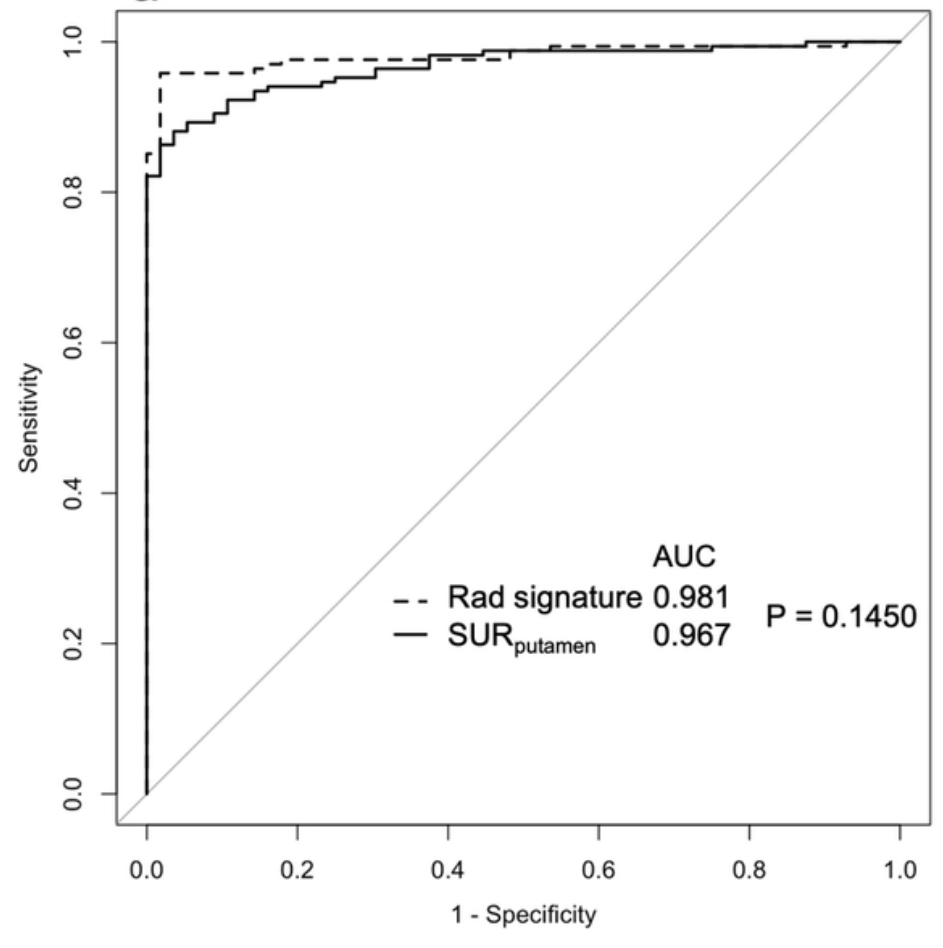

b

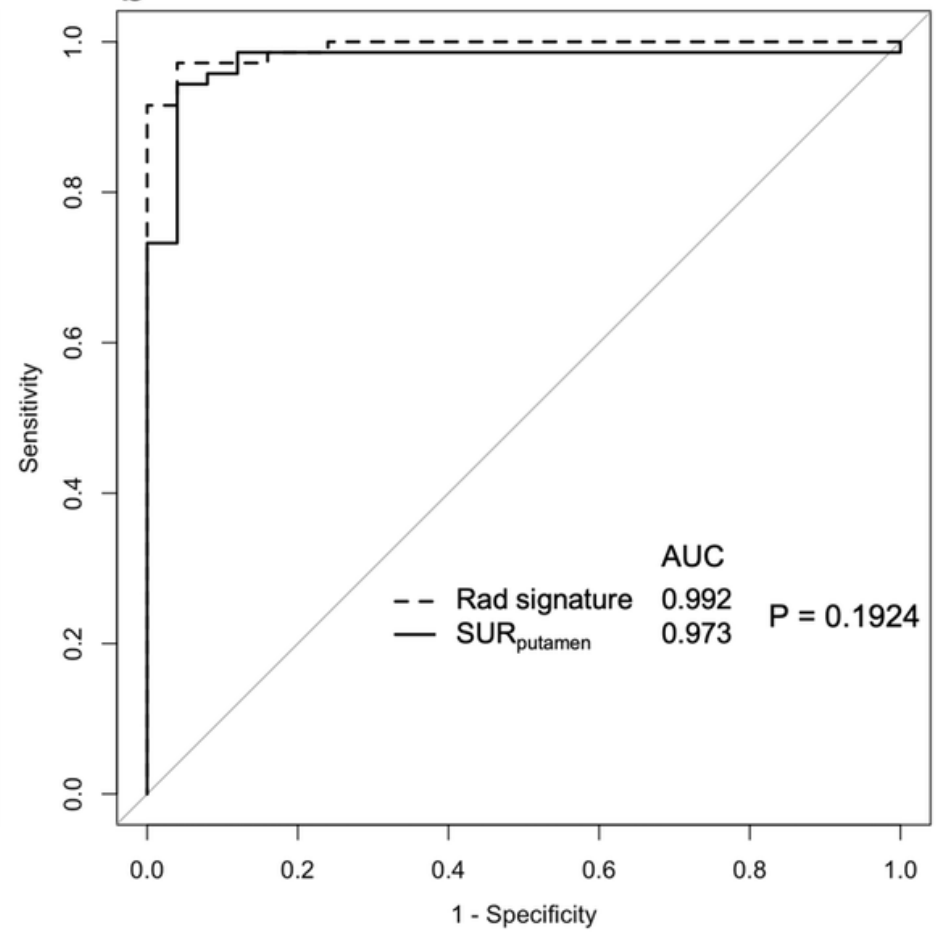

Figure 5

Comparison of receiver operating curves between radiomics signature and striatum uptake ratio of putamen

a The AUCs for the training set of the radiomics signature and SUR putamen $_{\text {were }} 0.981$ ( $95 \% \mathrm{Cl}, 0.965-$ $0.997)$ and $0.967(95 \% \mathrm{Cl}, 0.947-0.987)$, respectively $(P=0.1450)$. bTthe AUCs for the test set of the radiomics signature and SUR putamen were $0.992(95 \% \mathrm{Cl}, 0.981-1.00)$ and $0.973(95 \% \mathrm{Cl}, 0.939-1.00)$, respectively $(P=0.1924)$.

$A U C$ area under the curve, $\mathrm{Cl}$ confidence interval, SUR striatum uptake ratio 